\title{
Massive MIMO Communication Strategy Using Polarization Diversity For Industrial Scenarios
}

\author{
Frédéric Challita ${ }^{1}$, Pierre Laly ${ }^{1}$, Marwan Yusuf ${ }^{2}$, Emmeric Tanghe $^{2}$, Wout Joseph ${ }^{2}$, Martine Liénard ${ }^{1}$, Davy P. \\ Gaillot $^{1}$, Pierre Degauque ${ }^{1}$
}

\begin{abstract}
In this paper, a polarization diversity technique applied to massive MIMO in industrial environment is proposed. The main idea is to use dual-polarized elements at the fixed array and to adequately choose their polarization for each communication channel between this array and a given user. For the same number of antenna ports, such a configuration allows decreasing by a factor 2 the geometrical size of the array and thus minimizing the spatial non-stationarity over the array. Performances of this approach are evaluated in terms of sum-rate capacity assuming either a maximum ratio transmitter or a zero forcing precoding, and are based on channel matrices measured at $3.5 \mathrm{GHz}$ in an industrial hall.
\end{abstract}

Index Terms-Index Terms-Massive MIMO, Industry 4.0, Rx Spatial Correlation, Polarization Diversity.

\section{INTRODUCTION}

$\mathbf{I}$ $\mathrm{N}$ the frame of the 4 th industrial revolution also referred to as Industry 4.0 [1], [2], machine-to-machine (M2M) communication [3] becomes a critical topic. Some works have investigated the radio channel in industrial environments, but most of them refer to Single Input Single Output (SISO) links. For example, in [3], the authors present measurement results at $5.85 \mathrm{GHz}$ for communication between industrial robots. In [4], the polarization characteristics of specular and dense multipath components in a large industrial hall at $1.35 \mathrm{GHz}$ using RiMAX estimator are presented. Ultrawideband measurements were also performed from 3.1 to 10.6 $\mathrm{GHz}$ and statistical models were established [5]. Path loss, shadowing measurements and capacity analysis for industrial indoor environments are presented in [6] for three different industrial environments at 900, 1600, and $2450 \mathrm{MHz}$. However, to ensure communication with large numbers of sensors or user equipments (UEs) while satisfying requirements in terms of connectivity, reliability and low latency, MultipleInput Multiple-Output (MIMO) and more specifically massive MIMO techniques could be a promising solution. At the fixed base station (BS), massive MIMO consists in deploying a large antenna array with many radiating element on the order of 100 or more. Such an array also helps alleviating small-scale fading effects and allows decreasing inter-user

This work was funded through the OS4 SMARTIES research program by the ELSAT2020 project co-financed by the European Union with the European Regional Development Fund, the French state and Hauts de France Region.

F. Challita, P. Laly, D. P. Gaillot, M. Liénard and Pierre Degauque are with the University of Lille, IEMN, Bâtiment P3,Villeneuve d'Ascq, 59655 FR e-mail: frederic.challita@univ-lille.fr.

Emmeric Tanghe, Marwan Yusuf and Wout Joseph are with Ghent University/IMEC INTEC-WAVES 9052 Gent e-mail: wout.joseph@UGent.be

IEEE, Vol. xx, No. xx, October 2019 correlation [7], [8], [9]. As an example, massive MIMO system model for Industrial Internet of Things is presented in [10] and performance of different linear processing techniques is investigated. Nonetheless, massive MIMO measurements in industrial scenarios are quite scarce. In a previous work [11], polarimetric channel measurements of a Multi-User (MU) Multiple-Input Single-Output (MISO) setup for an indoor industrial scenario are presented. The center frequency and the bandwidth of analysis are equal to $3.5 \mathrm{GHz}$ and 80 $\mathrm{MHz}$, respectively, the number of array elements being 100 . Propagation characteristics and system-oriented metrics such as sum-rate capacity with maximum-ratio transmitter (MRT) precoder have been studied assuming vertically $(\mathrm{V})$ polarized antennas at the UEs, acting as receiving antennas (Rx), while all elements of the transmitting array (Tx) are either horizontally $(\mathrm{H})$ or $\mathrm{V}$ polarized. It was shown that when all Tx-Rx antennas are co-polarized (co-polar), high correlation between Rx antennas, mainly occurring in Line-of-Sight (LOS) configuration, gives rise to a strong decrease of the sum-rate capacity of the channels compared to an identicallyand-independent-distributed (i.i.d.) channel. To cope with this problem, a possible solution is to use cross polarization (Xpolar) transmission, all $\mathrm{Tx}$ array elements being $\mathrm{H}$ polarized. This was motivated by prior experimental results showing that, in this case, correlation decreases. Despite power loss at Rx due to X-polar conditions, it was shown in [11] that channel capacity, with MRT precoding, is improved especially in strong LOS scenario.

In this paper, we extend this previous study by proposing a novel communication scheme using simultaneously co- and $\mathrm{X}$-polar channels, all array elements being dual-polarized. The basic concept is that a given link between Tx and an UE will be made either in co- or X-polar conditions, depending on the correlation between this UE and its neighbors. This approach could allow finding the best compromise between additional path loss for X-polar channels and decrease of the correlation between nearby users. Another significant interest of using dual-polarized elements is that, with the same number of antenna ports, the size of the Tx array is reduced, avoiding non-wide sense stationary characteristics of the channels.

The proposed strategy will be evaluated using measured channels at $3.5 \mathrm{GHz}$ in the same industrial hall as in [11], [12]. Different LOS and NLOS configurations have been considered, the fixed Tx array being a $10 \times 10$ uniform square array. This letter is organized as follows. In Section II, the configuration of the environment is first described, including the characteristics of the antennas and the measurement setup. 
Section III recalls the main channel characteristics in terms of Rx spatial correlation and X-polar discrimination (XPD). In Section IV, the polarization diversity scheme based on an array of dual-polarized elements is first described. Then, two algorithms are proposed to select whose $K_{\mathrm{v}}$ channels will be co-polar and whose $K_{\mathrm{h}}$ channels will be X-polar. Section V is devoted to the performance of this communication strategy considering two linear precoders, MRT or zero-forcing (ZF) and using, as criteria, the sum-rate capacity. Lastly, Section VI concludes this work.

\section{EXPERIMENTAL SETUP}

In this paper, emphasis being placed on downlink (DL), the terms UE or Rx will be used indifferently. The geometrical configuration of the hall, the positions of the antennas and the principle of the channel sounding are successively presented.

\section{A. Geometrical Configuration}

Measurements were carried out in a large $21 \times 77 \times 12$ $\mathrm{m}^{3}$ industrial hall in Technologiepark-Zwijnaarde, Belgium. It is dedicated for testing the robustness of concrete, the latter being also the dominant building material. A panoramic view of the hall seen from Tx is shown in Fig. 1 a). The scenario is a MU-MISO setup with $K=15$ distributed single-antenna (V polarized) UEs and whose positions are given in Fig. 1. b) by the numbered circles. More details concerning the environment can be found in [11]. The Rx positions have been selected to cover various configurations, i.e. LOS (positions 1-2 and 4-7) and NLOS (positions 3, 8-15).

The horizontal distance between $\mathrm{Tx}$ and the farthest $\mathrm{Rx}$ is $56 \mathrm{~m}$. The transfer matrices were measured by moving the $\mathrm{Rx}$ antenna to each selected position at constant height equal to $1.6 \mathrm{~m}$. The Tx array is a virtual square array with $M=100$ elements situated at a mean height of $6.5 \mathrm{~m}$, thus, overlooking the hall as seen in Fig. 1.a). The positioning system for moving the Tx antenna is controlled through a fiber optics link with a dedicated LabView program installed on a Windows PC. The inter-element spacing of the virtual array is $\lambda / 2$ at the center frequency of $3.5 \mathrm{GHz}$ resulting in a $38 \mathrm{~cm} \times 38 \mathrm{~cm}$ array.

\section{B. Radio Channel Sounding}

Tx and Rx are monopolar patch antennas with a bandwidth of $80 \mathrm{MHz}\left(S_{11}<-10 \mathrm{~dB}\right)$ around $3.5 \mathrm{GHz}$. They have half-power beamwidth of $80^{\circ}$, both in azimuth and elevation with a $7 \mathrm{dBi}$ gain. The $\mathrm{Tx}$ antenna is manually rotated to transmit either on $\mathrm{H}$ or $\mathrm{V}$ polarization, Rx being always $\mathrm{V}$ polarized. The wideband complex channel transfer function between an $\mathrm{Rx}$ at position $k$ and any Tx array element is given by the $S_{21}$ scattering parameter measured by a vector network analyzer (VNA - Agilent E5071C) over an $80 \mathrm{MHz}$ bandwidth. The sampling frequency being $97.7 \mathrm{kHz}$, the number $M_{f}$ of frequency points is 819 . The VNA was situated in the vicinity of Tx and optical fiber optics were deployed for connecting Rx to a port of the VNA through optical/radio frequency interfaces.

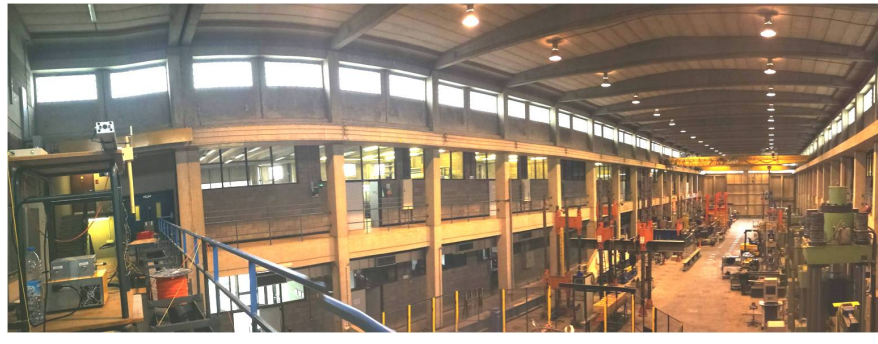

(a)

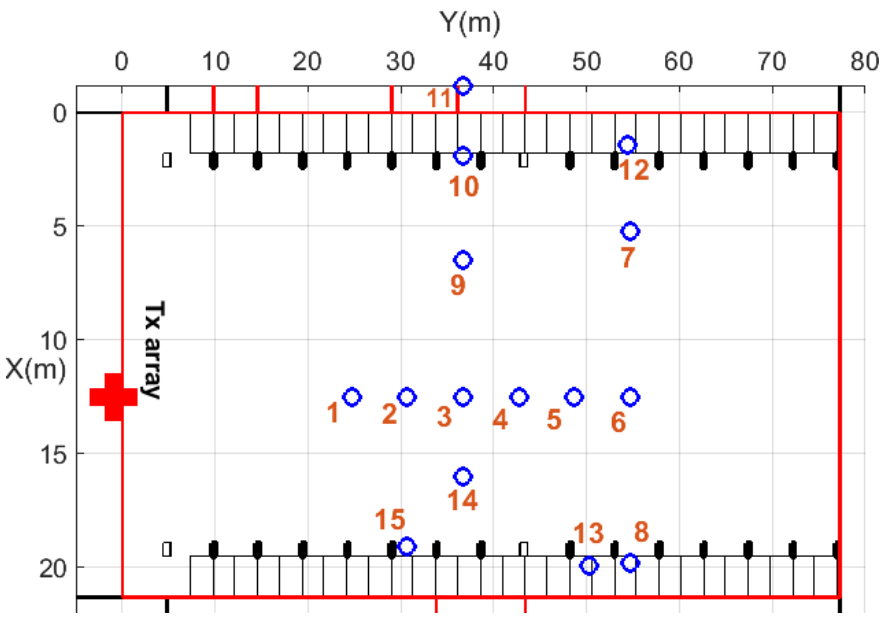

(b)

Fig. 1. a) Panoramic view of the industrial hall (Tx at the left side of the figure) and b) Top view showing the positions of Tx and those of the various UEs.

Considering all $K$ UEs and $M$ array elements, the full channel matrices $\mathbf{H}^{\mathrm{VV}}$ and $\mathbf{H}^{\mathrm{HV}}$ corresponding to co- and $\mathrm{X}$ polar channels, respectively, are determined. However, to make in the following, a fair comparison between the performances of the proposed approaches based on channels with different polarizations, these matrices must be normalized to take into account the additional path loss in X-polar conditions. At frequency $f$, the normalized matrices $\mathbf{H}_{n}$ are given by (1), where the subscript $F$ denotes the Froebinius norm.

$\mathbf{H}_{n}^{V V}(f)=\frac{\sqrt[2]{K M} \mathbf{H}^{V V}(f)}{\left\|\mathbf{H}^{V V}(f)\right\|_{F}} \quad \mathbf{H}_{n}^{H V}(f)=\frac{\sqrt[2]{K M} \mathbf{H}^{H V}(f)}{\left\|\mathbf{H}^{V V}(f)\right\|_{F}}$.

\section{Main Channel Characteristics}

\section{A. Rx Spatial Correlation}

Massive MIMO system advantages generally arise from channels orthogonality. For a MU scenario, the $K$ UEs channel orthogonality can be assessed from the $\mathbf{R}_{\mathrm{Rx}}$ spatial correlation matrix. For a given frequency, this matrix is given by (2) where the upper script $H$ means the Hermitian transpose.

$$
\mathbf{R}_{\mathrm{Rx}}(f)=\mathbf{E}\left\{\mathbf{H}_{n}(f) \mathbf{H}_{n}^{H}(f)\right\},
$$

In the following, the frequency points are used as independent observations and the final result will be computed as the average over frequencies. The spatial correlation between a couple $i$ and $j$ of UEs is denoted by $\rho_{i, j}$ and can be 
easily determined from the corresponding non-diagonal term of $\mathbf{R}_{\mathrm{Rx}}$. The median value of $\rho_{i, j}$ between a given UE and its three closest neighbors are given in Table I for LOS and NLOS scenarios. We observe that correlation between users is much greater in LOS condition than in NLOS scenarios. Furthermore, this correlation can be decreased if all Tx array elements are H-polarized, especially in LOS case, leading to full X-polar channels.

TABLE I

MEDIAN VALUE OF THE CORRELATION COEFFICIENT.

\begin{tabular}{|c|c|c|c|}
\hline \multicolumn{2}{|c|}{ LOS } & \multicolumn{2}{c|}{ NLOS } \\
\hline Co-polar & X-polar & Co-polar & X-polar \\
\hline 0.76 & 0.29 & 0.41 & 0.26 \\
\hline
\end{tabular}

From this point of view, XPD, defined as the ratio between average channel gain in co-polar mode to that in X-polar mode, is also a parameter that must be evaluated. The median XPD value for LOS and NLOS scenarios were found to be $15.5 \mathrm{~dB}$ and $10.1 \mathrm{~dB}$, respectively. To optimize the capacity of the link, the best compromise between high gain and low correlation must be found. Hence, we propose a novel communication scheme using simultaneously co- and X-polar channel. The choice of the channel polarization between $\mathrm{Tx}$ and a given UE depends on the spatial correlation between this UE and its neighbors.

\section{Communication Strategy Using Polarization DIVERSITY}

\section{A. Basic Principle}

Rather than to use the $M$ array elements either in $\mathrm{H}$ or $\mathrm{V}$ polarization, the approach developed in this section is to use $M / 2$ dual-polarized array elements instead of $M$ elements, all of them being either $\mathrm{H}$ or $\mathrm{V}$ polarized. The number of antenna ports $M$ is the same for both cases. The full massive MIMO channel is still defined by a $K \times M$ matrix, but the DL communication to the various UEs is made through either a VV or HV link (the first letter, $\mathrm{V}$ or $\mathrm{H}$, refers to the polarization of the Tx antennas). This is illustrated in Fig. 2 where (a) is the full VV channel (or HV channel) while (b) gives an example of polarization diversity scheme where $K_{\mathrm{v}}$ channels use a VV link and $K_{\mathrm{h}}$ an HV link.
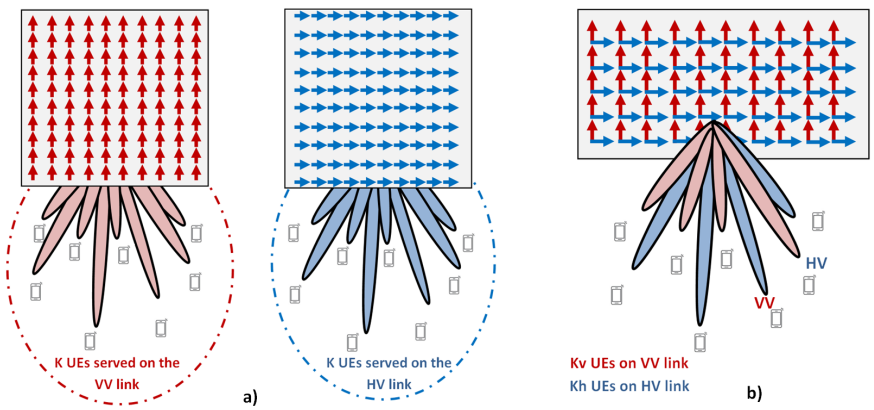

Fig. 2. Communication scheme with (a) Full co- or cross-polarized channel with $M=100$ and (b) Diversity scheme with $M / 2$ dual-polarized elements.
In this last case, the full channel matrix can be divided into two parts: (1) $\mathbf{H}_{c o} \in \mathbb{C}^{K_{v} \times M / 2}$ and (2) $\mathbf{H}_{\text {cross }} \in \mathbb{C}^{K_{h} \times M / 2}$. The proposed methods for allocating a VV or $\mathrm{HV}$ channel to a given UE is described in the next paragraph and their performance are then presented.

\section{B. UEs Polarization Allocation Algorithms}

The applied strategy relies on the $\mathrm{Rx}$ spatial correlation matrix defined in (2) in order to detect the pair $(i, j)$ of UEs which are highly correlated and to map channels $i$ and/or $j$ into orthogonal polarizations. Among the numerous possibilities to allocate a polarization to each channel, two strategies have been identified and investigated:

- Strategy 1 maps 2 channels, related to 2 UEs $i$ and $j$, on different polarizations $\mathrm{VV}$ and $\mathrm{HV}$ respectively, if and only if $\rho_{i, j}$ is greater or equal to a specified threshold $\rho_{t h}$. Otherwise, the VV co-polar for both UEs is kept in order to avoid additional power loss. A value of $\rho_{t h}=0.75$ has been chosen, a parametric study having shown that, above this value, the performance of the link decreases very rapidly. This strategy offers a trade-off between gain optimization and polarization diversity for highly correlated UEs. Since high spatial Rx correlation occurs in general for LOS scenarios, one can expect that the relative number of X-polar channels will be greater in this case than in NLOS scenarios. From the experimental channels measured in the hall, we get $K_{\mathrm{h}}=3$ and $K_{\mathrm{v}}=$ 12 , the number of LOS and NLOS channels being 6 and 9 , respectively.

- Strategy 2. In this approach, the number of co-and Xpolar channels are equal, $K_{\mathrm{h}}=K_{\mathrm{v}}=K / 2$, or nearly equal if $K$ is an odd number. The correlation coefficients $\rho_{i, j}$ are thus classified in decreasing order to deduce the channels which will be X-polarized. The purpose of this approach is to reduce the correlation as much as possible with no consideration on the total channel gain.

\section{Performance Of The Proposed Polarization DIVERSITY TECHNIQUES}

A practical and simple metric to quantify the performance of a massive MIMO system is the sum-rate capacity $C$ expressed in bits/s/Hz, related to the $K$ users. This metric will be used for evaluating the two proposed polarization allocation techniques with either MRT or ZF precoding.

\section{A. Analytical Expression Of The Sum-Rate Capacity}

Under perfect channel knowledge hypothesis, the achievable rate $C$ is a logarithmic function of the signal to interferenceplus-noise ratio (SINR). For a frequency $f$, it is given by:

$$
C=\sum_{k=1}^{K} \log _{2}\left(1+\operatorname{SINR}_{k}\right)
$$

where the $S I N R_{k}$ can be expressed as:

$$
\operatorname{SINR}_{k}=\frac{p_{k}\left|\mathbf{h}_{k} \mathbf{w}_{k}\right|^{2}}{\sum_{i=1, i \neq k}^{K} p_{i}\left|\mathbf{h}_{k} \mathbf{w}_{i}\right|^{2}+\sigma_{n}^{2}} .
$$


In (4), $p_{k}$ designates power allocated to the $k^{t h}$ user, $\mathbf{w}_{k}$ the corresponding precoding vector and $\sigma_{n}^{2}$ the noise variance. In the following examples, equal power allocation is assumed.

\section{B. Results Deduced From Measured Channel Matrices}

Curves in Fig. 3 (a) and (b) show the variation of $\mathrm{C}$ versus the average $S N R$ which would be obtained if all channels be VV co-polarized. Indeed, this configuration can be considered as a reference scenario to fully assess the efficiency of polarization diversity techniques. Results obtained if all channels are co-polar (VV) or X-polar (HV) can be compared in Fig. 3 with those obtained applying polarization allocation strategy 1 or 2 .

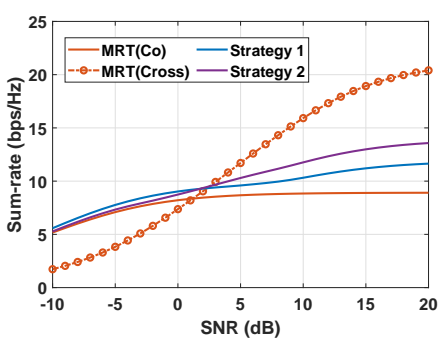

(a)

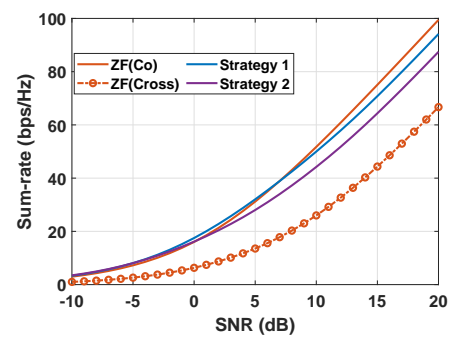

(b)
Fig. 3. Sum-rate capacity versus average $S N R$ either for full VV (Co) or full HV (Cross) polarization or for polarization diversity (Strategies 1 or 2). (a) MRT precoding, (b) ZF precoding.

For MRT and $S N R<0 \mathrm{~dB}$, both strategies give similar results compared to co-polar channels whereas at high $S N R$, performance of MRT in full X-polar channels remains the best configuration. Indeed, since MRT totally overlooks interference, the best results are obtained by minimizing UEs correlation and despite $\mathrm{Rx}$ power loss. We also note that strategy 2 is better than strategy 1 for the same reason. For $\mathrm{ZF}$ and whatever the $S N R$ value, strategy 1 and co-polarized channels result in similar sum-rate capacity values and are the best configurations. Indeed, since $\mathrm{ZF}$ aims at removing interference, an approach that focuses on maximizing channel gain gives the best result, as for full co-polar channels or polarization diversity with strategy 1 . Let us recall that with this strategy, only 3 channels among 15, are X-polar.

Lastly, comparison between curves in Fig. 3.a) and (b) shows that $\mathrm{ZF}$ is always better than MRT in terms of $C$ whatever the polarization scheme, as expected. To summarize these results, $C$ values are presented in Table $\mathrm{II}$ for the various polarization schemes: co-polar, X-polar and polarization diversity with strategies 1 and 2 . We assume an average $S N R$ equal to 10 or $20 \mathrm{~dB}$ in co-polar (VV).

We observe that the sum-rate capacities computed for strategy 1 and 2 are in the same order of magnitude, strategy 1 being slightly better with $\mathrm{ZF}$ precoding. The most interesting point is that, for $\mathrm{ZF}$, polarization diversity gives similar results compared to full co-polar channels but with a reduced array size (by a factor of 2). With MRT, $C$ values with polarization diversity are greater than those with full co-polarized channels but less than X-polarized channels.
TABLE II

SUM-RATE CAPACITY RESULTS FOR DIFFERENT SCHEMES OF CHANNEL POLARIZATION.

\begin{tabular}{|c|c|c|c|}
\hline \multicolumn{2}{|c|}{} & \multicolumn{2}{|c|}{$C$} \\
\hline Precoder & Scheme & $S N R=10 \mathrm{~dB}$ & $S N R=20 \mathrm{~dB}$ \\
\hline \multirow{4}{*}{ MRT } & Co & 9 & 9 \\
\cline { 2 - 4 } & Cross & 16 & 21 \\
\cline { 2 - 4 } & Strategy 1 & 10 & 12 \\
\cline { 2 - 4 } & Strategy 2 & 12 & 14 \\
\hline \multirow{4}{*}{ ZF } & Co & 52 & 100 \\
\cline { 2 - 4 } & Cross & 26 & 67 \\
\cline { 2 - 4 } & Strategy 1 & 50 & 96 \\
\cline { 2 - 4 } & Strategy 2 & 44 & 87 \\
\hline
\end{tabular}

In Fig. 4, the various curves highlight the influence of the number $M_{A P}$ of Tx antenna ports. To avoid confusion we have preferred to use the wording "antenna port" rather than radiating elements which could be either single or dualpolarized. The average $S N R$ of the co-polar VV configuration and used as a reference is chosen equal to $10 \mathrm{~dB}$.

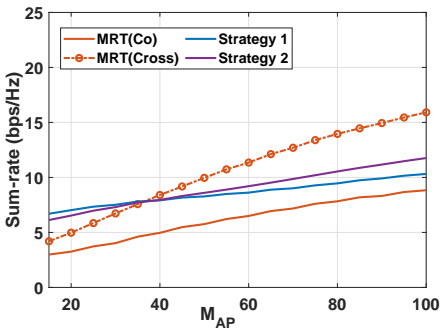

(a)

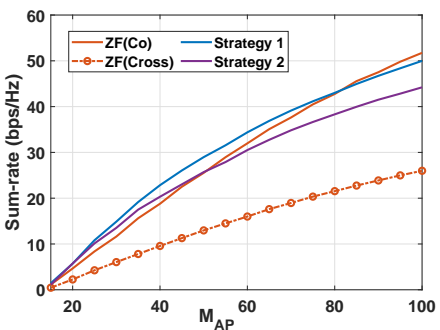

(b)
Fig. 4. Sum-rate capacity versus the number of antenna ports either for full VV (Co) or full HV (Cross) polarization or for polarization diversity (Strategy 1 or 2). (a) MRT precoding, (b) ZF precoding.

With MRT precoding, full X-polarization gives the best result only if $M_{A P}>40$. Conclusions on the performance of polarization diversity are the same as those given in the previous paragraphs when studying the influence of $S N R$.

\section{CONCLUSION}

This paper has investigated the possibility of using dualpolarized elements at the Tx array, the various UEs being always V-polarized. For the same number of antenna ports, results deduced from channel measurements in an industrial environment at $3.5 \mathrm{GHz}$, have shown that polarization diversity can reach comparable performances in terms of capacity than those obtained with full co-polar channels which is the best configuration if a ZF precoder is used. For MRT precoding, polarization diversity is better at low $S N R$ while at high $S N R$, its performances remain quite acceptable even if lower than those obtained if all channels are X-polar. Polarization diversity in an industrial environment seems thus a quite interesting solution for massive MIMO to decrease the geometrical size of the array and avoid spatial non-stationarity over this array. Future works include measurements in other industrial environments and additional tests of polarization strategies. 


\section{REFERENCES}

[1] S. K. Rao and R. Prasad, "Impact of 5G Technologies on Industry 4.0," Wirel. Pers. Commun., vol. 100, no. 1, pp. 145-159, May 2018. [Online]. Available: https://doi.org/10.1007/s11277-018-5615-7

[2] A. Varghese and D. Tandur, "Wireless requirements and challenges in Industry 4.0," in 2014 International Conference on Contemporary Computing and Informatics (IC3I), Nov 2014, pp. 634-638.

[3] B. Holfeld, D. Wieruch, L. Raschkowski, T. Wirth, C. Pallasch, W. Herfs, and C. Brecher, "Radio channel characterization at $5.85 \mathrm{GHz}$ for wireless M2M communication of industrial robots," in 2016 IEEE Wireless Communications and Networking Conference, April 2016, pp. 1-7.

[4] D. P. Gaillot, E. Tanghe, W. Joseph, P. Laly, V. C. Tran, M. Lienard, and L. Martens, "Polarization Properties of Specular and Dense Multipath Components in a Large Industrial Hall," IEEE Transactions on Antennas and Propagation, vol. 63, no. 7, pp. 3219-3228, July 2015.

[5] J. Karedal, S. Wyne, P. Almers, F. Tufvesson, and A. F. Molisch, "A Measurement-Based Statistical Model for Industrial Ultra-Wideband Channels," IEEE Transactions on Wireless Communications, vol. 6, no. 8, pp. 3028-3037, August 2007.

[6] E. Tanghe, W. Joseph, L. Verloock, L. Martens, H. Capoen, K. V. Herwegen, and W. Vantomme, "The industrial indoor channel: large-scale and temporal fading at 900, 2400, and $5200 \mathrm{MHz}$," IEEE Transactions on Wireless Communications, vol. 7, no. 7, pp. 2740-2751, July 2008.

[7] T. L. Marzetta, "Noncooperative Cellular Wireless with Unlimited Numbers of Base Station Antennas," IEEE Transactions on Wireless Communications, vol. 9, no. 11, pp. 3590-3600, November 2010.

[8] E. G. Larsson, O. Edfors, F. Tufvesson, and T. L. Marzetta, "Massive MIMO for next generation wireless systems," IEEE Communications Magazine, vol. 52, no. 2, pp. 186-195, February 2014.

[9] F. Rusek, D. Persson, B. K. Lau, E. G. Larsson, T. L. Marzetta, O. Edfors, and F. Tufvesson, "Scaling Up MIMO: Opportunities and Challenges with Very Large Arrays," IEEE Signal Processing Magazine, vol. 30, no. 1, pp. 40-60, Jan 2013.

[10] B. M. Lee and H. Yang, "Massive MIMO for Industrial Internet of Things in Cyber-Physical Systems," IEEE Transactions on Industrial Informatics, vol. 14, no. 6, pp. 2641-2652, June 2018.

[11] F. Challita, P. Laly, M. Liénard, D. P. Gaillot, E. Tanghe, M. Yusuf, and W. Joseph, "Impact of Polarization Diversity in Massive MIMO for Industry 4.0," in 2019 European Conference on Networks and Communications (EuCNC), June 2019, pp. 337-341.

[12] F. Challita, P. Laly, M. Yusuf, E. Tanghe, W. Joseph, P. Degauque, M. Liénard, and D. P. Gaillot, "Channel Correlation-based approach for feedback overhead reduction in massive MIMO," IEEE Antennas and Wireless Propagation Letters, pp. 1-1, 2019. 